\title{
Correlation Matrix Methods to Assess the Stirring Performance of Electromagnetic Reverberation Chambers
}

\author{
Gabriele Gradoni \\ School of Mathematical Sciences and George Green Institute for Electromagnetics Research, \\ University of Nottingham, University Park, Nottingham, United Kingdom NG7 2RD \\ Franco Moglie and Valter Mariani Primiani \\ Department of Information Engineering (DII), Università Politecnica delle Marche, \\ Ancona, Italy.
}

\begin{abstract}
The use of correlation matrices to evaluate the number of uncorrelated stirrer positions of electromagnetic reverberation chambers has widespread applications in electromagnetic compatibility. We present a review of recent methods based on multivariate correlation functions that relates statistical inhomogeneities in space (frequency) to the reduction of uncorrelated cavity configurations. Full wave finite-difference time domain simulations of an actual reverberation chamber are performed through an in-house parallel code. The efficiency of this code allows for capturing extensive inhomogeneous/anisotropic reverberation fields at frequencies close to the lowest usable frequency (LUF) of the chamber. The concept of effective independent position is revised in light of random sampling and a model-driven relation with the probability distribution of correlation matrix entries is used to take into account spatial (frequency) inhomogeneities. Driven by extensive simulation data, an empirical probability density function is found for the correlation matrix elements to be non-central $t$-student distributed with asymmetry increasing towards low frequencies.
\end{abstract}

\footnotetext{
${ }^{\text {th }}$ Financial support from EU Prace and Cineca (Bologna, Italy) is gratefully acknowledged. Email address: gabriele.gradoni@nottingham.ac.uk (Gabriele Gradoni)
} 


\section{Introduction}

Performances of a mode-stirred reverberation chamber (RC) in electromagnetic compatibility (EMC) applications are intimately related to the number of independent cavity realizations [1]. Unambiguous evaluation of the number of independent stirrer positions is still under investigation [38, 18]. Current approaches rely on the autocorrelation of fields sampled at single site inside the working volume (WV) of the RC. The autocorrelation function is also used to calculate the coherence time of the RC [44]. Previous investigators showed that, despite being inside the WV, by considering only one chamber site results in a high spatial variability of the autocorrelation coefficient $[3,10,40]$. This experimental evidence is of crucial importance as the commonly accepted notion of independence for a stirrer position is just defined by the $\rho_{e}=e^{-1}$ threshold crossing of the autocorrelation coefficient. Even though it brings about a simple and effective criterion, this metrics is empirical and borrows a certain amount of uncertainty in evaluating whether members of the cavity ensemble are strictly "independent" each other. To this regard, the threshold $\rho_{e}$ is typically associated with the concept of "uncorrelation" rather than "independence". Those observations naturally lead to the definition a correlation matrix having each entry defined by the correlation between two of the total $N_{s}$ stirrer positions. The number of entries of the so defined correlation matrix is given by $N_{s} \times N_{s}$. In a good RC, we expect a low value for many correlation matrix elements, i.e. many stirrer position pairs have "low correlation".

In this multivariate approach, an alternative way of evaluating the number of uncorrelated positions of mechanical mode-stirrers is based on the calculation of the correlation matrix through a grid of $N_{w}$ spatial points, selected among an arbitrary volume of the chamber [22]. An alternative application of the correlation matrix involves single-point measurements through a wide frequency range and therefore it leads to "uncorrelated frequencies" rather than spatial points [41]. This way proves more fast and efficient as populating spatial correlation matrix requires for a large amount of field/power measurements. Hybrid tech- 
niques can also be conceived based on the correlation matrix. Spatial point and frequency point data can be combined to check stirring performance.

In this work, we review existing multivariate strategies grounded on the correlation matrix that are important to assess RC performances. The typical framework we use to test different performance indicators is a reverberation chamber equipped with a "carousel" shape mechanical stirrer [36]. Being based on multi-spatial multi-frequency sampling, these indicators can be used in arbitrary complex dynamic electromagnetic environments. In particular, the mode stirrer can be either present as in RCs or absent as in wave chaotic billiards or enclosures $[17,6,23,43]$. Correlation based analysis of uncorrelated chamber states are useful to analyze the $\mathrm{RC}$ behavior in the transition between undermined and overloaded regimes, as well as to create finite sets of realizations behaving closely to ideal statistical ensembles. The strategies being used throughout the paper are focused on the evaluation of:

1. uncorrelated stirrer positions adopting a spatial correlation matrix (henceforth referred as "US-PM");

2. uncorrelated stirrer positions adopting a frequency correlation matrix (henceforth referred as "US-FM");

3. uncorrelated frequency steps adopting a spatial correlation matrix for a single stirrer position (henceforth referred as "UF-PM").

In any strategy we analyze the effect of varying the involved parameters, number and distance of frequency and spatial points, and we compare results to those obtained applying the 1-D circular autocorrelation method, as suggested by IEC standard [1] and applied on simulated $S_{21}$ parameters.

Finally, we use the correlation matrix to investigate:

4. the uncorrelated frequency steps adopting a stirrer position correlation matrix at a single spatial point (henceforth referred as "UF-SM");

5. uncorrelated spatial points adopting a stirrer position correlation matrix for each single frequency (henceforth referred as "UP-SM"); 


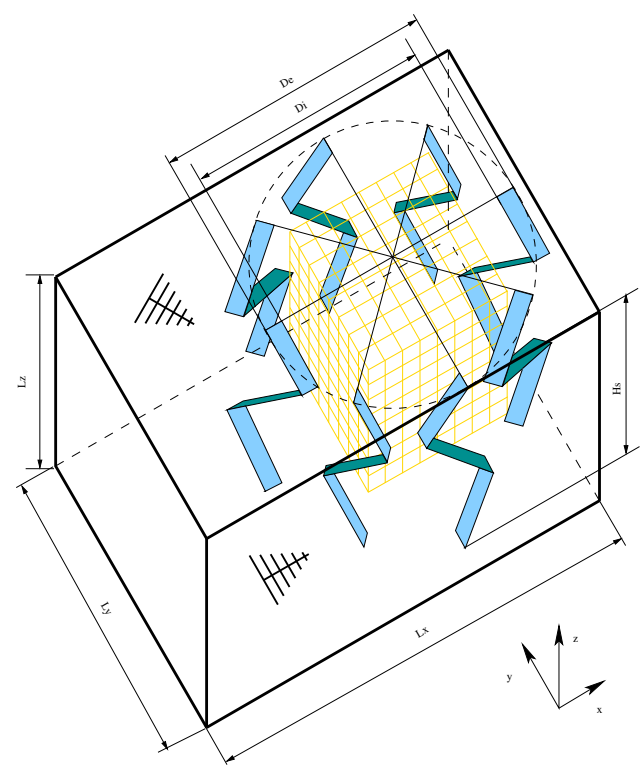

Figure 1: Geometry of the reverberation chamber (RC) equipped with a "carousel" stirring system. The simulated RC is that of Ancona EMC laboratory, it is $L_{x}=6 \mathrm{~m} \mathrm{long}, L_{y}=4 \mathrm{~m}$ wide and $L_{z}=2.5 \mathrm{~m}$ height. The vertical plates are $h=2.4 \mathrm{~m}$ high and they have a Z-folded shape. The rotating system describes a cylindrical volume whose base is centered in $x=4 \mathrm{~m}$ and $y=2 \mathrm{~m}$, the external diameter is $d_{e}=3.8 \mathrm{~m}$ and the internal diameter is $d_{i}=3.3 \mathrm{~m}$.

6. uncorrelated spatial points adopting a frequency correlation matrix (henceforth referred as "UP-FM").

\section{RC Performance Indicators}

The use of the circular autocorrelation function (ACF) [1] is well accepted for evaluating the number of statistically uncorrelated stirrer configurations, $N_{\text {eff }}$. More advanced methods based on the ACF have been proposed to assess the performances of mode-tuned reverberation chambers (MTRC) [28, 3]. Recently, the concept of multi-resolution for the construction of correlation matrices in MTRC has attracted the interest of the EMC community [13] where the MTRC performances are evaluated considering multiple space/frequency points, in contrast to the single space/frequency point approach adopted in the ACF method. 
We now illustrate the different multi-resolution methods proposed in the literature to estimate $N_{\text {eff }}$ from an ensemble of $N_{s}$ cavity realizations. A definition of uncorrelated stirrer positions based on a multivariate approach has been proposed in $[38,22]$. A correlation matrix has been defined in [22, Eqs. (1)-(6)]

$$
\underline{\underline{R}}_{s}=\left[\begin{array}{cccc}
\rho_{11} & \rho_{12} & \ldots & \rho_{1 N_{s}} \\
\rho_{21} & \rho_{22} & \ldots & \rho_{2 N_{s}} \\
\rho_{31} & \rho_{32} & \ldots & \rho_{3 N_{s}} \\
\vdots & \vdots & \vdots & \vdots \\
\rho_{N_{s} 1} & \rho_{N_{s} 2} & \ldots & \rho_{N_{s} N_{s}}
\end{array}\right],
$$

where $\rho_{i j}$ is the Pearson correlation coefficient between pairs calculated as detailed in [22, Eqs. (8) and (9)], and where $N_{s}$ is the total number of cavity realizations, e.g., the number of stirrer configurations in mechanical stirring. The number of multiple space/frequency points adopted in the calculation of individual Pearson coefficients is hereafter denoted with $M$. In [10], the number of uncorrelated positions are related to the number of independent eigenvalues of the correlation matrix $\underline{\underline{R}}_{s}$. Those elements are calculated as two-point correlation functions between two arbitrary points, and therefore require multi-resolved field sampling. This has been achieved, for example, through a "platform stirring" strategy [10]. From information theoretic arguments, it turns out that the number of independent eigenvalues is

$$
N_{\text {eff }}=\frac{\operatorname{Tr}^{2}\left[\underline{\underline{R}}_{s}\right]}{\operatorname{Tr}\left[\underline{\underline{R}}_{s}^{2}\right]},
$$

where $\operatorname{Tr}[\cdot]$ indicates the trace of a matrix. This perspective has the advantage of being threshold-less, i.e., the elements of $\underline{\underline{R}}_{s}$ do not need to be tested against the empirical $e^{-1}$ limit to achieve $N_{\text {eff }}$. Threshold-based approaches currently adopted in the IEC normative [1] rely on single point circular autocorrelation coefficient (CC) to evaluate $N_{\text {eff }}$. It has been shown that, with respect to the IEC method, the expression (2) underestimates $N_{\text {eff }}$.

A similar philosophy is adopted in [41] where the concern is the number of uncorrelated measurements rather than stirrer positions. The measurement 
correlation has been defined in terms of the maximum Renyì entropy, again based on the eigenvalues of the correlation matrix. In particular, given the maximum entropy (maxentr) achievable through $N_{s}$ measurements

$$
S_{2}=\log \left(N_{\text {eff }}\right)
$$

it is straightforward to have an estimation of the number of uncorrelated measurements reading

$$
N_{\text {eff }}=e^{S_{2}} .
$$

Since $S_{2}$ can be calculated from the eigenvalues $\lambda_{n}$ of $\underline{\underline{R}}_{s}$, denoted as $\lambda_{n}$, then

$$
N_{\text {eff }}=\frac{\left(\sum_{n=1}^{N_{s}} \lambda_{n}\right)^{2}}{\sum_{n=1}^{N_{s}} \lambda_{n}^{2}} .
$$

The analogies between between (5) and (2) are many, and essentially they constitute the same formula achieved through two diverse approaches [8]. The advantage of (5) is that the correlation matrix can be populated through a frequency scanning, i.e., a frequency sampling would lead to $\underline{\underline{R}}_{f}$ as in [41], rather than the spatial scanning, leading to $\underline{\underline{R}}_{s}$. It is worth remarking that the estimator (2), used in different versions in [41] and [10], depends on the sample length, and the estimated number of uncorrelated positions $N_{\text {eff }}$ is given by [ 8 , Eq. (14)]

$$
\hat{N}_{\text {eff }}=\frac{N_{\text {eff }}}{1+\frac{N_{\text {eff }}}{M}},
$$

where, again, $M$ indicates the number of multiple space/frequency points adopted in the calculation of individual Pearson coefficients, or sample length in $[8$, Sec. 2.b.], and $\hat{N}_{\text {eff }}$ is the sample estimate of $N_{\text {eff }}$. The bias expression in (6) converges to $N_{\text {eff }}$ for a relatively high number of degrees of freedom $M$, i.e., $M \gg N_{\text {eff }}$. A proof of (6) can be obtained by noting that the correlation matrix can be expanded in true principal components (PC) (see [8] for more details). It has been indeed demonstrated that the $\mathrm{RC}$ field admits $\mathrm{PC}$ decomposition of the stirring process in empirical (time-domain) modes, called as "eigenstirrings" [5]. Inherently, (6) can be also used to perform a systematic correction 
of the estimates if we make use of (2) to evaluate the number of effective stirrer positions with a few finite difference time domain (FDTD) lattice points [22].

Based on an entropic perspective, we can use (5) to carry out the calculation of uncorrelated stirrer positions through either $\underline{\underline{R}}_{s}$ or $\underline{\underline{R}}_{f}$. It has been already acknowledged that the production of entropy from scattering processes over finite regions of space is important to evaluate the degrees of freedom of a complex EM system [9]. A study of the time-domain entropy generation in RC has been performed in [19], through numerical FDTD simulations of an actual $\mathrm{RC}$, and the linear increase at early time followed by a saturation to the maxentr limit of entropy at late time

$$
S_{2} \approx \begin{cases}\sigma t \log \left(N_{\mathrm{eff}}\right), & t<t_{b} \\ \log \left(N_{\mathrm{eff}}\right), & t>t_{b}\end{cases}
$$

where $\sigma$ is the growth rate, $t_{b}$ is a build-up time of which an estimate is given in [26], was observed to saturate at the maxentr limit (3) which converges to the high-frequency estimate. This behavior is typical of multi-component (many-body) complex systems and has been observed in quantum many-body systems operating in chaotic regime [42]. Very recently, a similar result has been demonstrated in the context of time-dependent Hamiltonians with Floquet instabilities, where the entanglement entropy of a quantum system grows in time with a rate determined by the Lyapunov instability exponent [7]. Therefore, in the high frequency asymptotic regime, where the $\mathrm{RC}$ field mixing can be imagined to take place through the interference of several plane waves following classical ray trajectories, the so-called semiclassical regime, the result in $[7$, Theorem 2] for a wave system supports the hypothesis that

$$
\sigma \approx \sum_{n=1}^{L_{+}} \Lambda_{n}
$$

where $L_{+}$is the number of positive (classical) Lyapunov exponents $\Lambda_{n}$. The connection between (7) and (8) deserves further modeling effort in order to be clarified. This is a non-trivial task that involves the connection between entropy of the random field underpinned by interference, and classical ray dynamics 
associated with the wave system subject to instability and chaos.

A step forward has been carried out in [39] through the evaluation of uncorrelated pairs in the correlation matrix. The method is based on graph theory to estimate the number of uncorrelated stirrer positions. A graph is developed where each node corresponds to a stirrer position, and an arc is formed when the correlation between two positions $(i, j)$ satisfies the cutoff condition [1]

$$
r_{i j}=\left[\underline{\underline{R}}_{s}\right]_{i j} \leq \mathrm{e}^{-1}\left[1-\frac{7.22}{\left(N_{s}^{2}\right)^{0.64}}\right] \text {. }
$$

Equation (9) is an extension of the correlation threshold $\rho_{e}$ based on sampling theory. The Authors then use standard algorithms to search for the so-called "maximum clique" of the graph, and identifies it as the maximum number of uncorrelated positions. The result is a graphical method to count the number of uncorrelated positions through a direct test of uncorrelated node pairs. The method in $[38,3]$ relies on a threshold, while the methods proposed in $[10,40]$ do not rely on it, i.e., they can be classified as threshold-less.

The presence of a threshold should not be taken necessarily as a limitation. Actually, its physical significance will become clear in a random sampling perspective, where we imagine that correlation matrix $\underline{\underline{R}}_{s, f}$ entries are realizations of a unique random correlation coefficient that fluctuates under space/frequency inhomogeneity.

In the multivariate approach, we introduce a generalized threshold-based procedure to estimate the number of uncorrelated positions, by exploiting information of an extended reverberation (sub) space rather than a single point. The generality of the method resides in the fact that the sampling over multiple spatial points/frequencies has been extended to an arbitrary reverberant subspace independently on the a-priori definition of the $\mathrm{WV}$, and beyond the orientation of the device under test. The mathematical procedure is detailed in [22]. The number of uncorrelated stirrer positions comes from counting the number of elements in $\underline{\underline{R}}_{s, f}$ that are below the threshold (9). Each element has the meaning of a correlation between two cavity sub-spaces (calculated through a discrete space/frequency lattice [20] generated from two different stirrer con- 
figurations. More precisely, this number is obtained by taking account of the symmetry properties of $\underline{\underline{R}}_{s, f}$, and of the fact that $r_{i i}=1$, hence

$$
N_{\mathrm{eff}}=\frac{N_{s}^{2}}{\#[\underline{\underline{R}} s, f>r \underline{\underline{1}}]},
$$

where \#[.] is the counting operator, $\underline{\underline{1}}$ is the a square matrix of dimension $N_{s}$, where all elements are 1 . When two stirrers are used in an $\mathrm{RC}$, we follow the procedure described in [35]. Here, the case involving $\underline{\underline{R}}_{s}$ corresponds to US-PM, while the case involving $\underline{\underline{R}}_{f}$ corresponds to US-FM.

Interestingly, besides stirrer positions, our method can be used to evaluate the number of uncorrelated spatial points given measurements from a set of stirrer positions (US-SM, UP-FM). Also, the number of uncorrelated working frequencies can be estimated from stir sequences (UF-SM), or spatial lattice sampling (UF-SM).

In all cases, the threshold given in (10) is adopted.

\section{Full-wave simulations and results}

We now evaluate the number of uncorrelated stirrer positions of an actual RC simulated by an in-house numerical code. In particular, we focus on the stirring performances of the "carousel" stirrer previously studied in [36]. Figure 1 shows the detailed geometry: the stirrer consists of equispaced metallic $z$-folded blades. Its rotation describes a cylindrical volume which bounds the uniformity volume of the chamber, i.e., the working volume (WV).

According to the method discussed in Section 2, we do not restrict our investigations to an a-priori defined working volume, further requiring a calibration protocol such as that describe in $[1, \mathrm{~A} .5]$.

Full wave FDTD simulations of the RC have been recently used by other investigators [33, 2, 14]. An in-house FDTD code, optimized for BlueGene computer architecture [32, 37], is used to perform a full wave simulation of the Ancona's RC. The EM fields can then be sampled over a dense grid of spatial points (sampling lattice), for an arbitrary number of stirrer positions [22]. 
The $\mathrm{RC}$ is then discretized in $201 \times 134 \times 84$ cubic cells with a side of 30 $\mathrm{mm}$, the FDTD time step is $\Delta t=50 \mathrm{ps}$, and the number of iterations necessary to analyze the entire structure is around 200000. In the code, the chamber walls, the carousel blades, and the two antennas are considered made of ideal conductors. Volumetric air losses are inserted to account for whole losses in the real chamber operating in our laboratory. The adoption of these equivalent volumetric losses allow to speed up the computation time, and a provides reliable numerical results at the same time [34]. Frequency data are obtained by fast Fourier transform (FFT). Figure 1 shows the sampling lattice (gold solid line grid) adopted in the simulations, where the $N_{w}$ points are $0.15 \mathrm{~m}$ equispaced.

The discrete (total or Cartesian) field $E_{\left(m_{i}, m_{j}, m_{k}\right)}^{(\tau)}$ is picked up at $N_{w}$ spatial points $\left(m_{i} \Delta x, m_{j} \Delta y, m_{k} \Delta z\right)$ of the sampling grid, for each stir state $\tau_{i}=i \Delta \theta$, $i=1, \ldots, N_{s}$, with $\Delta \theta$ angular stirrer step, and $N_{s}$ total number of stirrer positions considered in the analysis. In discretizing the continuous stirring, we assumed that the "uncorrelation angle" of the carousel stirrer is greater than $\Delta \theta$. The correlation is then computed by using $N_{p}$ field values. In particular, for the total electric field we have $N_{p}=N_{w}$, while for the three separate Cartesian components we have $N_{p}=3 N_{w}$.

The RC is simulated for 512 (equispaced) stirrer angles, and for each angle the three Cartesian field components are computed in a grid of $8 \times 8 \times 8=512$. To reduce the burden of storing very big files, the values computed by the FFT subroutine were reduced to 2622 equidistant frequency points in the range $0.2-$ 1.0 GHz. The memory required to store all the data is 189 GBytes.

\subsection{Quality factor and coherence bandwidth}

We start our analysis by computing the quality factor of the simulated RC in the investigated frequency range. An average quality factor (Q) can be derived directly from simulated scattering data by using the formula [25]

$$
Q=\frac{16 \pi^{2} V\left\langle\left|S_{21}\right|^{2}\right\rangle}{\eta_{T x} \eta_{R x} \lambda^{3}\left(1-\left|\left\langle S_{11}\right\rangle\right|^{2}\right)},
$$




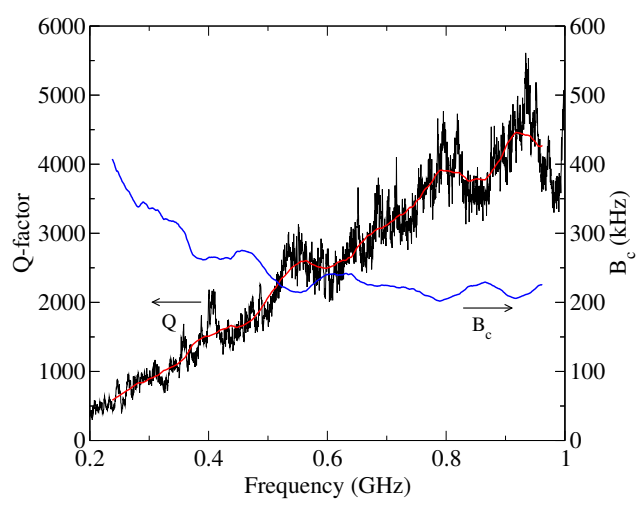

Figure 2: Simulated Q-factor and coherence bandwidth.

where $V$ is the RC volume, $\lambda$ the free-space wavelength, $S_{21}$ the complex scattering transmission, and $S_{11}$ the complex scattering reflection coefficient, $\eta_{T x}$ and $\eta_{R x}$ the transmitting and receiving antenna efficiency respectively. With reference to Figure 1, the transmitting antenna is in the point $(1.53,1.31,0.89) \mathrm{m}$ whereas the receiving one is in $(0.71,3.19,1.41) \mathrm{m}$.

Regarding the efficiencies, we assumed ideal conductors in the antenna simulations, $\eta_{T x}=\eta_{R x}=1$. Pertaining the mismatching factor, we separated the incident and reflected signals in the transmission line feeding the antennas, so we got the realistic scattering parameters. Only in the case of lossless and load matched antennas, $\eta_{T x}=\eta_{R x}=1$, and $\left\langle S_{11}\right\rangle=\left\langle S_{22}\right\rangle=0$. An accurate estimate of $Q$ allows for recovering the RC coherence bandwidth $B_{c}[11,16,27]$

$$
B_{c}=\frac{f}{\langle Q\rangle} .
$$

Figure 2 shows the simulated quality factor and the corresponding coherence bandwidth. It is worth noticing that for working frequencies $f>350 \mathrm{MHz}$, the $\mathrm{RC}$ coherence bandwidth becomes lower than the sampling frequency step we use, which is $\Delta f \approx 305 \mathrm{kHz}$. This regime corresponds to high-quality factor modes accompanied by a high modal density which is predicted by the Weyl law [4]. Despite in high frequency regime, we expect for spectral rigidity and avoided level crossing to cause overlapping between non-degenerate modes [17, 6, 12]. 


\subsection{Uncorrelated stirrer positions}

Referring to the notation of Section 1, we compute $N_{\text {eff }}$ either through sampling grid of $N_{w}$ spatial points, i.e., the case 1) US-PM, and through a frequency scanning over $N_{f}$ frequency points, i.e., the case 2) US-FM. Figure 3 shows $N_{\text {eff }}$ as calculated with $N_{w}=512$ and $N_{f}=512$. The same Figure also reports $N_{\text {eff }}$ computed through the standard, i.e., circular correlation (CC), technique as described in the IEC standard [1]. It is worth noticing that the US-PM method underestimates $N_{\text {eff }}$ with respect to the CC method. This happens also varying the number of grid spatial points [20] and the number of frequency points [21]. Conversely, the US-FM method overestimates $N_{\text {eff }}$. Again, the sampling frequency step is greater than or equal to $B_{c}$. The effect of bandwidth variation was investigated in [22], where a lower number of frequency points were adopted to build up the correlation matrix.

Interestingly, the curve related to US-FM saturates when the cavity coherence bandwidth reaches the sampling frequency, viz.,

$$
B_{c} \approx \Delta f=305 \mathrm{kHz}
$$

Below this frequency, evaluating uncorrelated positions with this strategy mean sampling over $B_{c}$, as shown in Figure 2 , whence $B_{c}<\Delta f$ results in $N_{\text {eff }} \leq N_{s}$.

Finally, the same Figure reports also the computation applying the thresholdless method (2). As expected, the threshold method gives a lower number of uncorrelated stirrer angles. In fact, equation (2) would return all the 512 positions as uncorrelated only if all off-diagonal elements of the correlation matrix were null, above a certain frequency. This is impossible in practice, because the electric field correlation coefficient between two spatial points in the chamber is zero only for particular values of distance and frequency, and asymptotically tends to zero for an infinite distance $[24,15]$.

\subsection{Uncorrelated frequency points}

In those RC applications, where the electronic stirring method is thus employed, it is important to know whether the frequency steps are uncorrelated 


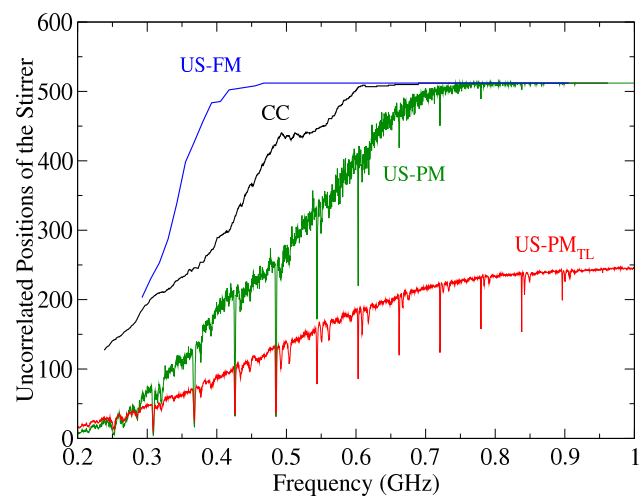

Figure 3: Simulated uncorrelated stirrer positions, for a correlation matrix of grid points (US$\mathrm{PM}$ ) and of frequency points (US-FM). Results applying the IEC standard method are also reported $(\mathrm{CC})$. The trace $\left(\mathrm{US}-\mathrm{PM}_{\mathrm{TL}}\right.$ ) shows the results obtained applying the thresholdless method (2).

or not. Figure 4 shows the number of uncorrelated frequencies as calculated in the UF-PM strategy. It is worth pointing out two interesting features. Adopting the total field $|E|$, the frequency points become correlated below $400 \mathrm{MHz}$, where the frequency step is lower than the chamber $B_{c}$. On the other hand, the single rectangular component gives a lower number of frequency uncorrelation points. Conversely, there is no difference when the uncorrelated stirrer positions are evaluated by $|E|$ or by $E_{x, y, z}[20]$, i.e. in the US-PM strategy. Results refer to a fixed stirrer angle. The gray area in Figure 4 shows the spreading of the uncorrelated frequency points for 16 stirrer positions. This indirectly quantifies the uncertainty of the estimation. Figure 4 shows a very limited spreading.

The determination of uncorrelated frequency number can also be done populating the correlation matrix with the field values computed in a single chamber point by rotating the stirrer (UF-SM). Figure 5 shows the simulated number of uncorrelated frequencies. A similar behavior to the case UF-PM is observed. The gray area of Figure 5 shows a larger uncertainty than the one in Figure 4.

Apart this, it can be noted that populating the matrix by field samples collected in many spatial points (Figure 4) the number of uncorrelated frequencies is a little bit lower than the case when the matrix is populated by field samples collected for many stirrer positions (Figure 5). This discrepancy is evident 


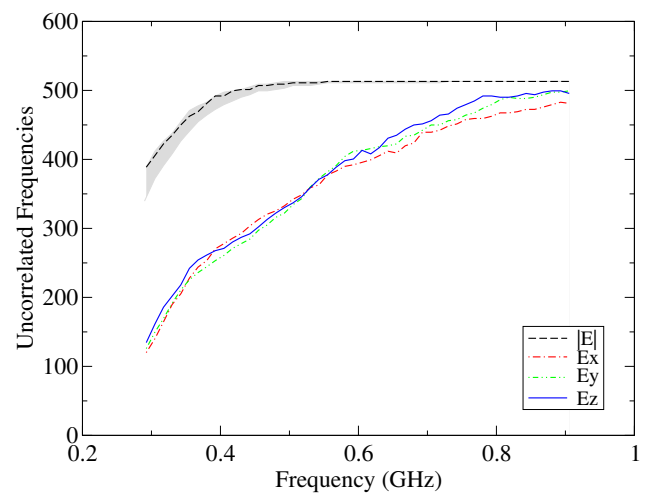

Figure 4: Simulated uncorrelated frequency points for a correlation matrix of grid points (UF-PM). Values computed by using each component and the magnitude of the electric field are reported. The gray area is the spread for 16 stirrer angles.

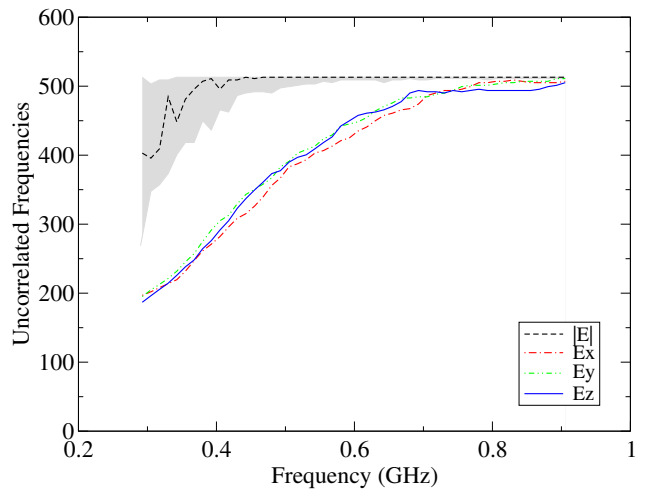

Figure 5: Simulated uncorrelated frequency points for a correlation matrix of stirrer angles (UF-SM). The gray area is the spread for all the 512 grid points. 
up to about $650 \mathrm{MHz}$ in adopting the single Cartesian field components, and it needs further investigation after analysing the uncorrelation degree of both stirrer positions and spatial points, Section 4.

\subsection{Random stir sampling}

The definition (10) has a straightforward physical meaning: the number of independent positions can be evaluated as the number of stirring configurations having acceptable spatial dispersion of the (Cartesian or total) field variance. Therefore, given a sampling subspace, it is possible to construct a two-position correlation function over a discretized, i.e., tuned, stirrer rotation. The theoretical prediction of $N_{\text {eff }}$ based on (10) is not an easy task.

In a very general fashion, we could also treat $r_{i} j$ as a random variable through $\underline{\underline{R}}_{s, f}$. In this perspective, its probability distribution ${ }^{1}$ would be regulated by the space/frequency sampling. Inherently, a randomized sampling over continuous stirrer rotations is more realistic and would naturally call for a statistical treatment of $r_{i j}$, though in principle also in deterministic sampling it is possible to linearize $\underline{\underline{R}}_{s, f}$, order its elements $r_{i j}$, and calculate the probability density function (PDF) of the correlation as a distribution of its values. Therefore, also $N_{\text {eff }}$ becomes a random variable with probability density function $f_{N_{\text {eff }}}\left(n_{e f f}\right)$. Exploiting the addition theorem in probability, we can turn the probability of the counting operator into a union of probabilities of the single element $f_{R}(r<\bar{r})$. Obviously, the ability of obtaining many single uncorrelated elements $r=r_{i j} \leq \bar{r}$ depends on the stirring parameters such as structure geometry, location, and dimensions. The evaluation of the stirring efficiency can be performed deterministically by full-wave numerical simulations or measurements on actual structures. Our perspective calls for a statistical approach to the problem: instead of a pure deterministic evaluation, it would be useful to introduce a probability $f_{R}(r<\bar{r})$ of the mode-stirrer to make two arbitrary realizations uncorrelated.

\footnotetext{
${ }^{1}$ meaning frequency of value occurrence in the Laplace sense
} 
Furthermore, counting $n_{\text {eff }}$ positions in $\mathcal{N}_{s}$ elements means having $n_{\text {eff }}$ successes with probability $f_{R}(r<\bar{r})$ in $\mathcal{N}_{s}$ trials, that is given by the Bernoulli (or binomial) distribution. The use of this probability distribution function requires assuming that the entries of the correlation matrix (1), in our case the Pearson correlation coefficients, are statistically uncorrelated. Further assuming a deterministic threshold of $\bar{r}$, yields

$$
\begin{aligned}
f_{N_{\text {eff }}}\left(n_{e f f}, \bar{r}\right)= & \left(\begin{array}{c}
n_{e f f} \\
\mathcal{N}_{s}
\end{array}\right)\left[f_{R}(r<\bar{r})\right]^{n_{e f f}} \\
& {\left[1-f_{R}(r<\bar{r})\right]^{\mathcal{N}_{s}-n_{e f f}}, }
\end{aligned}
$$

where

$$
\left(\begin{array}{c}
n_{e f f} \\
\mathcal{N}_{s}
\end{array}\right)=\frac{n_{e f f} !}{\mathcal{N}_{s} !\left(n_{e f f}-\mathcal{N}_{s}\right) !},
$$

is the Newton binomial, which accounts for the fact that we have $\mathcal{N}_{s}$ elements that can be either above or below the threshold $\bar{r}$, and the effective number of considered pairs of chamber configurations is given by half the number of off-diagonal elements of the correlation matrix, viz.,

$$
\mathcal{N}_{s}=\frac{N_{s}\left(N_{s}-1\right)}{2},
$$

and the event probability is actually a cumulative distribution function (CDF)

$$
F_{R}(\bar{r})=f_{R}(r<\bar{r})=\int_{0}^{\bar{r}} f_{R}(r) d r .
$$

Further physical considerations would be involved in presence of lattice autocorrelation. In this paper we treat the case of uncorrelated sampling space/frequency lattice. Once the distribution of $n_{\text {eff }}$ has been obtained, it is possible to obtain a robust estimation of the number of independent stirrer positions by calculating the following averaging

$$
\bar{N}_{\text {eff }}(\bar{r})=\int \delta\left(n_{e f f}-n\right) f_{N_{\text {eff }}}(n, \bar{r}) d n,
$$

which is given by

$$
\bar{N}_{\text {eff }}(\bar{r})=\mathcal{N}_{s} F_{R}(\bar{r})
$$

The challenge is now to derive the probability distribution of the correlation matrix entries $f_{R}(r)$, and to understanding its dependence on chamber and 


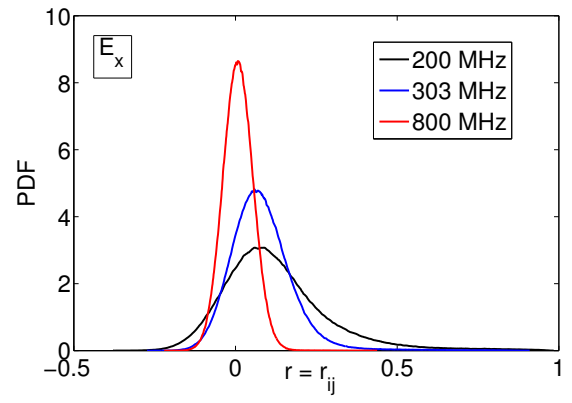

Figure 6: Probability density function of the off-diagonal element of correlation matrix for $\left|E_{x}\right|$ at low $(200 \mathrm{MHz})$, intermediate $(303 \mathrm{MHz})$, and high $(800 \mathrm{MHz})$ frequency, for a spatial grid $13 \times 13 \times 11$ of the US-PM strategy.

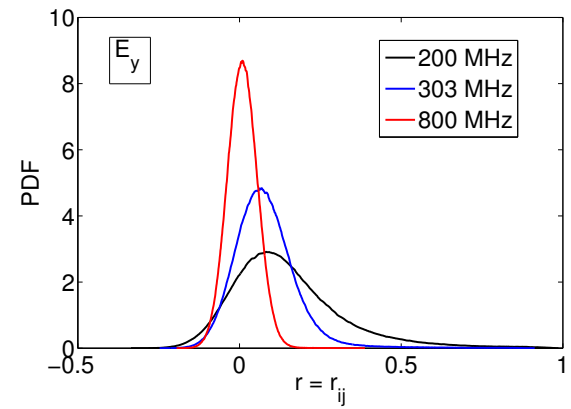

Figure 7: Probability density function of the off-diagonal element of correlation matrix for $\left|E_{y}\right|$ at low $(200 \mathrm{MHz})$, intermediate $(303 \mathrm{MHz})$, and high $(800 \mathrm{MHz})$ frequency, for a spatial grid $13 \times 13 \times 11$ of the US-PM strategy.

stirrer parameters $[35,31]$ such as chamber volume and losses. Here, we present empirical distributions of the correlation function resulting from a numerical (FDTD) model of the reverberation chamber. It is worth to be noticed that this model-driven distribution captures the fluctuation of the correlation in space or frequency, depending on the ensemble of cavity realizations chosen a priori for the analysis. Individual Pearson correlation elements are also subject to local fluctuations given by the sampling involved in the stirring process.

The PDF of $f_{R}(r)$ is reported in Figure 6 for the correlation of the Cartesian field $\left|E_{x}\right|$, as obtained through the US-PM strategy. Similar distributions are obtained for the components $E_{y}$ and $E_{z}$. Those are shown in Figure 7 and Figure 8. Interestingly, the $\mathrm{PDF}$ of $\left|E_{x}\right|,\left|E_{y}\right|$, and $\left|E_{z}\right|$ has non-central $t$ - 


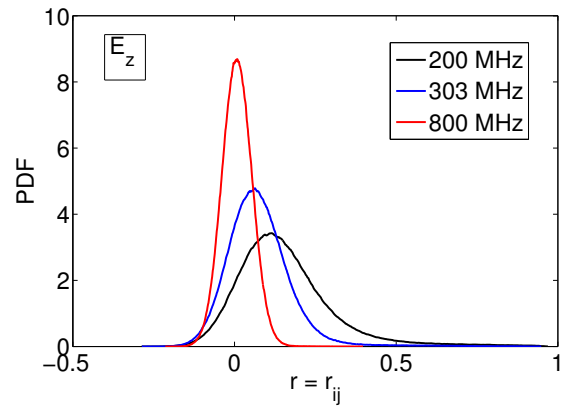

Figure 8: Probability density function of the off-diagonal element of correlation matrix for $\left|E_{z}\right|$ at low $(200 \mathrm{MHz})$, intermediate $(303 \mathrm{MHz})$, and high $(800 \mathrm{MHz})$ frequency, for a spatial grid $13 \times 13 \times 11$ of the US-PM strategy.

student profile. Also, observations confirm isotropic behavior and ergodicity over the investigated frequency range. Furthermore, it looks had to get rid of any residual correlation, whence the importance of fixing a threshold, and the genesis of the uncorrelation seems to be related to the positive tail. In this perspective, the presence of a threshold is of key importance as it looks that non-central behavior of the correlation PDF at low frequencies creates a fat tail in the region $r_{i j}>e^{-1}$. The presence of statistical inhomogeneities and anisotropies create non-central $t$-student probability distributions with large skewness. This fact can be exploited formally to make the rule in (19) more explicit by using the expression of the mean value of a non-central $t$-distribution [30]

$$
\bar{N}_{\text {eff }}(\bar{r}) \approx K \mathcal{N}_{s}\left\{\Phi(-\mu)+\frac{1}{2}\left[\sum_{j=0}^{\infty} p_{j} I_{n}\left(j+\frac{1}{2}, \frac{\nu}{2}\right)+q_{j} I_{n}\left(j+1, \frac{\nu}{2}\right)\right]\right\} .
$$

where $K$ represents a normalization factor, $\mu$ the non-centrality parameter, $\nu$ the number of degrees of freedom of the $t$-student distribution, $\Phi$ is the CDF of the standard normal distribution, $I_{n}$ is the regularized incomplete beta function 
and

$$
\begin{aligned}
n & =\frac{\bar{r}^{2}}{\bar{r}^{2}+\nu}, \\
p_{j} & =\frac{1}{j !} \exp \left(-\frac{\mu^{2}}{2}\right)\left(\frac{\mu^{2}}{2}\right)^{j}, \\
q_{j} & =\frac{\mu}{\sqrt{2} \Gamma\left(j+\frac{3}{2}\right)} \exp \left(-\frac{\mu^{2}}{2}\right)\left(\frac{\mu^{2}}{2}\right)^{j},
\end{aligned}
$$

in which it expected an inverse proportionality of $\mu$ and $\nu$ on frequency. Therefore, further studies are necessary to relate the number of correlated realization pairs to the $t$-student non-centrality and degrees of freedom. Furthermore, the sampling effect of the finite number of realization pairs can be accounted for by using results on effective sample size achieved in [29] as well as the Fisher distribution as described in [22].

\section{Uncorrelated spatial points}

Figure 9 shows the simulated number of uncorrelated grid points for a correlation matrix of stirrer angles (UP-SM). Again, when computed through the total electric field magnitude, this number exhibits higher values with respect to the single components. By using the rectangular components, the adopted grid points are uncorrelated above $800 \mathrm{MHz}$, where the point distance is $0.4 \lambda$. This result is close to the expected spatial correlation length of $0.5 \lambda$. By using the total field, the adopted grid points are uncorrelated above $650 \mathrm{MHz}$, where the point distance is $0.325 \lambda$. From a practical point of view, there is a residual correlation among spatial points in the lower frequency range, that results in less efficient stirring action if compared to the rotating stirrer, for the considered chamber of course. More precisely, comparing Figure 3 and Figure 9, we can observe that the 512 stirrer angles are more uncorrelated respect to the 512 spatial points, by applying the same threshold-based method. In fact, the rotating stirrer exhibits a higher number of uncorrelated positions respect to that given by the positioning stirring (spatial points). The higher correlation that affects the spatial points might be responsible of the discrepancy observed in 


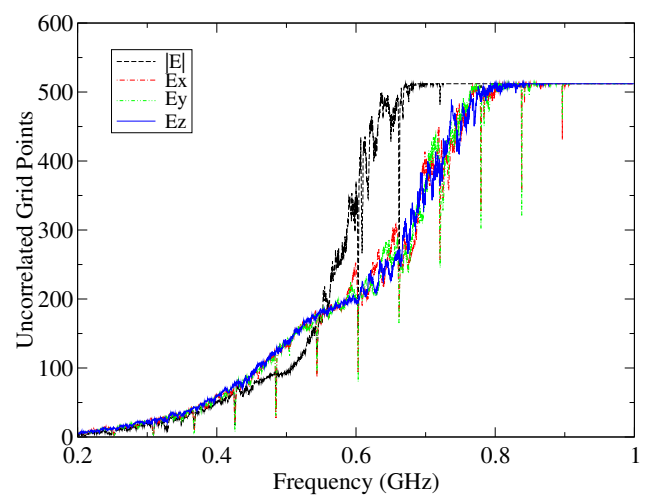

Figure 9: Simulated uncorrelated grid points for a correlation matrix of stirrer positions (UP$\mathrm{SM})$. Values computed by using each component and the magnitude of the electric field are reported.

Section 3.3 between Figure 4 and Figure 5. The correlation matrix adopted for Figure 4 has more correlated elements respect to the elements of that used for Figure 5, so resulting in an underestimation of the uncorrelated frequency points below $0.65 \mathrm{GHz}$. The effect of the number of stirrer positions used to compute the correlation matrix is reported in Figure 10. When the number of positions is large enough, the results converge to the same values, while decreasing the number of positions gives rise to lower values. This means that the correlation must be computed over a sufficiently large data ensemble. The curve behavior around $0.65 \mathrm{GHz}$, shows a large peak. In order to explain it, we considered the scattering parameters reported in Figure 11. The $S_{11}$ shows a reflection increment around $0.65 \mathrm{GHz}$. This is due to the resonance between antenna and stirrer at the particular location used throughout the experiments. This peak is not present in the $S_{21}$ and in the field spatial distribution. Moreover, regular frequency peaks where correlation increases appear. These peaks are also visible in the US-PM case, see Figure 3, and investigated in [20]. They do not depend on the adopted strategy, but on the RC geometry. In particular, peak distance corresponds to a value for which the $\mathrm{RC}$ dimension along the stirrer rotation axis is $0.5 \lambda$, which is $60 \mathrm{MHz}$ for this $\mathrm{RC}$.

Figure 12 shows the simulated number of uncorrelated grid points for the 


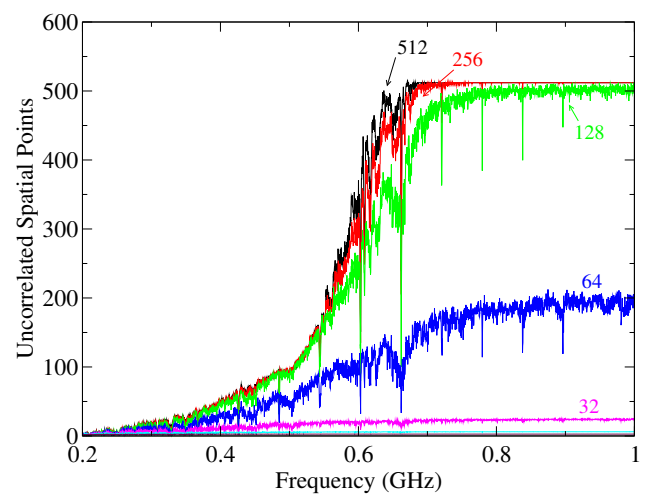

Figure 10: Simulated uncorrelated grid points as function of the number of stirrer positions used in the computation of the correlation matrix (UP-SM). The $|E|$ was used.

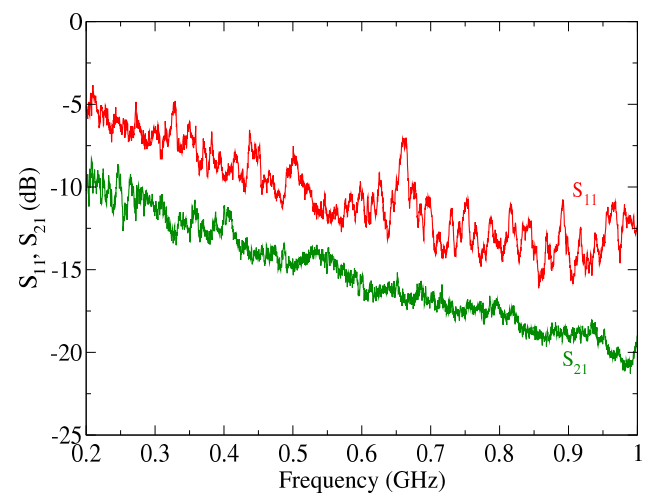

Figure 11: Simulated scattering parameters. 


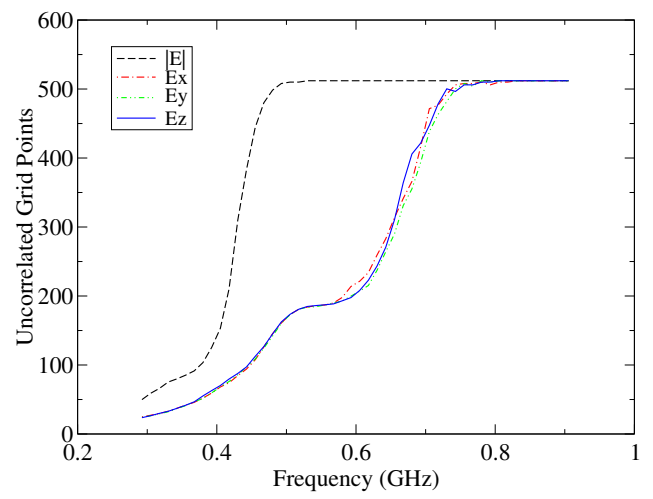

Figure 12: Simulated uncorrelated grid points for a correlation matrix of frequency points (UP-FM). Values computed by using each component and the magnitude of the electric field are reported.

strategy UP-FM. At each working frequency, we compute the correlation coefficients using a set of $N_{f}$ frequency points, which defines the frequency stirring bandwidth $B_{w}$. When we use $N_{f}=512, B_{w}=156 \mathrm{MHz}$. The effect of the number of frequency points adopted for the computation of the uncorrelated grid point number is reported in Figure 13. The frequency points where the correlation is calculated are the same. All the frequency points are equidistant, therefore reducing $N_{f}$ has the effect of decreasing $B_{w}$. When the number of frequency points is large enough, the results converge. Decreasing $N_{f}$, a reduction of the number of uncorrelated points is observed. In the FM strategies, peaks where uncorrelation decreases are not visible due to the intrinsic frequency averaging of the method.

\section{Conclusions}

We have compared existing methods to estimate the number of uncorrelated stirrer positions through the correlation matrix of multivariate stir traces. An actual reverberation chamber has been simulated through a full-wave, finitedifference time-domain, parallel code. Spatial and frequency multi-point sampling of stir traces offers a large scale picture of the field distributions within reverberation chambers. A threshold is introduced to count the uncorrelated 


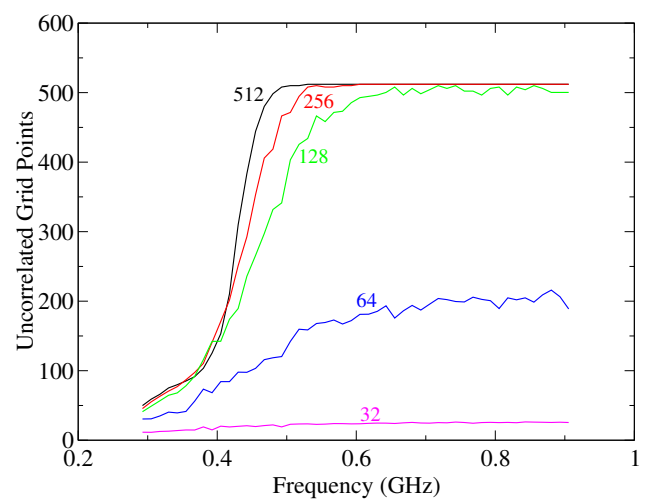

Figure 13: Simulated uncorrelated grid points as function of the number of frequency points used in the computation of the correlation matrix (UP-FM). The $|E|$ was used.

pairs of stirrer positions. Inherently, we observed that the classical autocorrelation method overestimates the uncorrelated blade positions. Estimation of uncorrelated frequencies and spatial grid points is also possible, which is of interest in frequency and spatial stirring techniques often adopted to change the chamber states. We noted an overestimation of uncorrelated pairs if frequency samples are used. The use of Cartesian field components underestimate the uncorrelated frequency and space points. Conversely, uncorrelated stirrer positions are not effected by this choice. In general, a sufficiently large date ensemble is necessary to correctly compute the correlation matrix elements. Finally, the probability density function of the correlation matrix elements is found to be non-central $t$-student distributed with asymmetry increasing towards the low frequency of chamber operation. This study suggests a physics-based study to relate the statistics of the effective number of uncorrelated stirrer positions with the non-centrality and degree-of-freedom parameters of the t-student distribution.

[1] 61000-4-21, I. S. I., Apr. 2011. Electromagnetic compatibility (EMC) - Part 4-21: Testing and measurement techniques - Reverberation chamber test methods. Geneva, Switzerland, 2nd Edition.

[2] Adardour, A., Andrieu, G., Reineix, A., Apr. 2014. On the low-frequency 
optimization of reverberation chambers. IEEE Transactions on Electromagnetic Compatibility 56 (2), 266-275.

[3] Amador, E., Lemoine, C., Besnier, P., 2011. Numerical study of spatial correlation in reverberation chamber. Electronics Letters 47 (24), 13191320.

[4] Arnaut, L. R., Nov. 2001. Operation of electromagnetic reverberation chambers with wave diffractors at relatively low frequencies. IEEE Transactions on Electromagnetic Compatibility 43 (4), 637-653.

[5] Arnaut, L. R., Feb. 2014. Optimizing low-frequency mode stirring performance using principal component analysis. IEEE Transactions on Electromagnetic Compatibility 56 (1), 3-14.

[6] Barthélemy, J., Legrand, O., Mortessagne, F., Jan 2005. Complete s matrix in a microwave cavity at room temperature. Phys. Rev. E 71, 016205.

[7] Bianchi, E., Hackl, L., Yokomizo, N., 2017. Linear growth of the entanglement entropy and the Kolmogorov-Sinai rate. arXiv:1709.00427 [hep-th].

[8] Bretherton, C. S., Widmann, M., Dymnikov, V. P., Wallace, J. M., Blade, I., Jul. 1990. The effective number of spatial degrees of freedom of a timevarying field. Journal of Climate 12 (7), 1990-2009.

[9] Bucci, O. M., Franceschetti, G., Jul. 1989. On the degrees of freedom of scattered fields. IEEE Trans. Antennas Propag. 37 (7), 918-926.

[10] Chen, X., Dec. 2012. On independent platform sample number for reverberation chamber measurements. IEEE Transactions on Electromagnetic Compatibility 54 (6), 1306-1309.

[11] Chen, X., Kildal, P.-S., Orlenius, C., Carlsson, J., 2009. Channel sounding of loaded reverberation chamber for over-the-air testing of wireless devices: Coherence bandwidth versus average mode bandwidth and delay spread. IEEE Antennas Wireless Propag. Lett. 8, 678-681. 
[12] Cozza, A., May 2011. The role of losses in the definition of the overmoded condition for reverberation chambers and their statistics. IEEE Transactions on Electromagnetic Compatibility 53 (2), 296-307.

[13] Cozza, A., Sep. 2011. A skeptic's view of unstirred components. In: EMC Europe 2011 York. York, UK, pp. 174-179.

[14] Cui, Y., Wei, G., Wang, S., Fan, L., Zhao, Y., Jun. 2014. Fast analysis of reverberation chamber using FDTD method and matrix pencil method with new criterion for determining the number of exponentially damped sinusoids. IEEE Transactions on Electromagnetic Compatibility 56 (3), 510519.

[15] De Doncker, P., 2003. Spatial correlation functions for fields in threedimensional Rayleigh channels. Progress In Electromagnetics Research 40, $55-69$.

[16] Delangre, O., De Doncker, P., Lienard, M., Degauque, P., 2008. Delay spread and coherence bandwidth in reverberation chamber. Electronics Letters $44(5), 328-329$.

[17] Dörr, U., Stöckmann, H.-J., Barth, M., Kuhl, U., Feb 1998. Scarred and chaotic field distributions in a three-dimensional sinai-microwave resonator. Phys. Rev. Lett. 80, 1030-1033.

[18] Gradoni, G., Bastianelli, L., Mariani Primiani, V., Moglie, F., Aug. 2015. Uncorrelated frequency steps in a reverberation chamber: A multivariate approach. In: Proc. IEEE Int. Symp. Electromagn. Compat. (EMC). Dresden, Germany, pp. 558-562.

[19] Gradoni, G., Mariani Primiani, V., Moglie, F., Sep. 2012. Reverberation chamber as a statistical relaxation process: Entropy analysis and fast time domain simulations. In: 2012 International Symposium on Electromagnetic Compatibility (EMC EUROPE). Rome, Italy, pp. 1-6. 
[20] Gradoni, G., Mariani Primiani, V., Moglie, F., Aug. 2013. Carousel stirrer efficiency evaluation by a volumetric lattice-based correlation matrix. In: 2013 IEEE International Symposium on Electromagnetic Compatibility (EMC). Denver, CO, USA, pp. 819-824.

[21] Gradoni, G., Mariani Primiani, V., Moglie, F., Sep. 2013. Determination of the reverberation chamber stirrer uncorrelated positions by means of the spatial and frequency correlation matrix. In: 2013 International Symposium on Electromagnetic Compatibility (EMC EUROPE). Brugge Belgium, pp. $425-430$.

[22] Gradoni, G., Mariani Primiani, V., Moglie, F., 2013. Reverberation chamber as a multivariate process: FDTD evaluation of correlation matrix and independent positions. Progress In Electromagnetics Research 133, $217-$ 234.

[23] Gros, J. B., Legrand, O., Mortessagne, F., Richalot, E., Selemani, K., 2014. Universal behaviour of a wave chaos based electromagnetic reverberation chamber. Wave Motion 51 (4), 664 - 672, innovations in Wave Modelling.

[24] Hill, D. A., Feb. 1995. Spatial correlation function for fields in a reverberation chamber. IEEE Transactions on Electromagnetic Compatibility 37 (1), $138-$.

[25] Hill, D. A., Ma, M. T., Ondrejka, A. R., Riddle, B. F., Crawford, M. L., Johnk, R. T., Aug. 1994. Aperture excitation of electrically large, lossy cavities. IEEE Transactions on Electromagnetic Compatibility 36 (3), 169178.

[26] Holloway, C. L., Shah, H. A., Pirkl, R. J., Remley, K. A., Hill, D. A., Ladbury, J., Aug 2012. Early time behavior in reverberation chambers and its effect on the relationships between coherence bandwidth, chamber decay time, rms delay spread, and the chamber buildup time. IEEE Transactions on Electromagnetic Compatibility 54 (4), 714-725. 
[27] Holloway, C. L., Shah, H. A., Pirkl, R. J., Remley, K. A., Hill, D. A., Ladbury, J., Aug. 2012. Early time behavior in reverberation chambers and its effect on the relationships between coherence bandwidth, chamber decay time, RMS delay spread, and the chamber buildup time. IEEE Transactions on Electromagnetic Compatibility 54 (4), 714-725.

[28] Lemoine, C., Besnier, P., Drissi, M., Nov. 2007. Investigation of reverberation chamber measurements through high-power goodness-of-fit tests. IEEE Transactions on Electromagnetic Compatibility 49 (4), 745-755.

[29] Lemoine, C., Besnier, P., Drissi, M., May 2008. Estimating the effective sample size to select independent measurements in a reverberation chamber. IEEE Transactions on Electromagnetic Compatibility 50 (2), 227-236.

[30] Lenth, R. V., 1989. Algorithm as 243: Cumulative distribution function of the non-central t distribution. Journal of the Royal Statistical Society. Series C (Applied Statistics) 38 (1), 185-189.

[31] Mariani Primiani, V., Moglie, F., Jun. 2012. Numerical simulation of reverberation chamber parameters affecting the received power statistics. IEEE Transactions on Electromagnetic Compatibility 54 (3), 522-532.

[32] Mariani Primiani, V., Moglie, F., Apr. 2014. Reverberation chamber performance varying the position of the stirrer rotation axis. IEEE Transactions on Electromagnetic Compatibility 56 (2), 486-489.

[33] Mengue, S., Richalot, E., Picon, O., May 2008. Comparison between different criteria for evaluating reverberation chamber functioning using a 3-D FDTD algorithm. IEEE Transactions on Electromagnetic Compatibility 50 (2), 237-245.

[34] Moglie, F., Bastianelli, L., Mariani Primiani, V., Jun. 2016. Reliable finitedifference time-domain simulations of reverberation chambers by using equivalent volumetric losses. IEEE Transactions on Electromagnetic Compatibility 58 (3), 653-660. 
[35] Moglie, F., Mariani Primiani, V., May 2011. Analysis of the independent positions of reverberation chamber stirrers as a function of their operating conditions. IEEE Transactions on Electromagnetic Compatibility 53 (2), 288-295.

[36] Moglie, F., Mariani Primiani, V., Apr. 2012. Numerical analysis of a new location for the working volume inside a reverberation chamber. IEEE Transactions on Electromagnetic Compatibility 54 (2), 238-245.

[37] Moglie, F., Mariani Primiani, V., Aug. 2014. Numerical simulations of field values, wave impedance, and received power inside a nonideal reverberation chamber. IEEE Transactions on Electromagnetic Compatibility 56 (4), 994-997.

[38] Pfennig, S., Krauthäuser, H. G., Sep. 2012. A general method for determining the number of independent stirrer positions in reverberation chambers. In: 2012 International Symposium on Electromagnetic Compatibility (EMC EUROPE). pp. 1-6.

[39] Pfennig, S., Krauthäuser, H. G., Sep. 2013. Comparison of methods for determining the number of independent stirrer positions in reverberation chambers. In: 2013 International Symposium on Electromagnetic Compatibility (EMC EUROPE). Brugge Belgium, pp. 431-436.

[40] Pirkl, R. J., Aug. 2013. Spatial autocovariances of scattering parameters measured in a lossy reverberation chamber. IEEE Transactions on Electromagnetic Compatibility 55 (4), 671-682.

[41] Pirkl, R. J., Remley, K. A., Lötbäck Patané, C. S., Jun. 2012. Reverberation chamber measurement correlation. IEEE Transactions on Electromagnetic Compatibility 54 (3), 533-545.

[42] Santos, L. F., Borgonovi, F., Izrailev, F. M., Mar. 2012. Chaos and statistical relaxation in quantum systems of interacting particles. Phys. Rev. Lett. 108, 094102. 
[43] Selemani, K., Gros, J. B., Richalot, E., Legrand, O., Picon, O., Mortessagne, F., Feb 2015. Comparison of reverberation chamber shapes inspired from chaotic cavities. IEEE Transactions on Electromagnetic Compatibility $57(1), 3-11$.

[44] Sorrentino, A., Ferrara, G., Migliaccio, M., Oct. 2009. On the coherence time control of a continuous mode stirred reverberating chamber. IEEE Trans. Antennas Propag. 57 (10), 3372-3374. 